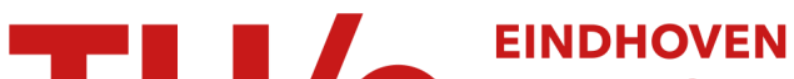 UNIVERSITY OF TECHNOLOGY
}

\section{Metastable neon collisions : anisotropy and scattering length}

\author{
Citation for published version (APA): \\ Mogendorff, V. P., Vredenbregt, E. J. D., Verhaar, B. J., \& Beijerinck, H. C. W. (2004). Metastable neon \\ collisions : anisotropy and scattering length. Physical Review A : Atomic, Molecular and Optical Physics, 69(1), \\ 012706-1/8. [012706]. https://doi.org/10.1103/PhysRevA.69.012706, \\ https://doi.org/10.1103/PhysRevA.69.012706
}

DOI:

10.1103/PhysRevA.69.012706

10.1103/PhysRevA.69.012706

Document status and date:

Published: 01/01/2004

\section{Document Version:}

Publisher's PDF, also known as Version of Record (includes final page, issue and volume numbers)

\section{Please check the document version of this publication:}

- A submitted manuscript is the version of the article upon submission and before peer-review. There can be important differences between the submitted version and the official published version of record. People interested in the research are advised to contact the author for the final version of the publication, or visit the $\mathrm{DOI}$ to the publisher's website.

- The final author version and the galley proof are versions of the publication after peer review.

- The final published version features the final layout of the paper including the volume, issue and page numbers.

Link to publication

\section{General rights}

Copyright and moral rights for the publications made accessible in the public portal are retained by the authors and/or other copyright owners and it is a condition of accessing publications that users recognise and abide by the legal requirements associated with these rights.

- Users may download and print one copy of any publication from the public portal for the purpose of private study or research.

- You may not further distribute the material or use it for any profit-making activity or commercial gain

- You may freely distribute the URL identifying the publication in the public portal.

If the publication is distributed under the terms of Article 25fa of the Dutch Copyright Act, indicated by the "Taverne" license above, please follow below link for the End User Agreement:

www.tue.nl/taverne

Take down policy

If you believe that this document breaches copyright please contact us at:

openaccess@tue.nl

providing details and we will investigate your claim. 


\title{
Metastable neon collisions: Anisotropy and scattering length
}

\author{
V. P. Mogendorff, * E. J. D. Vredenbregt, B. J. Verhaar, and H. C. W. Beijerinck \\ Physics Department, Eindhoven University of Technology, P.O. Box 513, 5600 MB Eindhoven, The Netherlands
}

(Received 9 September 2003; revised manuscript received 24 October 2003; published 15 January 2004)

\begin{abstract}
In this paper we investigate the effective scattering length $a$ of spin-polarized $\mathrm{Ne}^{*}$. Due to its anisotropic electrostatic interaction, its scattering length is determined by five interaction potentials instead of one, even in the spin-polarized case, a unique property among the Bose condensed species and candidates. Because the interaction potentials of $\mathrm{Ne}^{*}$ are not known accurately enough to predict the value of the scattering length, we investigate the behavior of $a$ as a function of the five phase integrals $\Phi_{\Omega}$ corresponding to the five interaction potentials. We find that the scattering length has five resonances instead of only one and cannot be described by a simple gas-kinetic approach or the degenerate internal state (DIS) approximation. However, the probability for finding a positive or large value of the scattering length is not enhanced compared to the single-potential case. We find that the induced dipole-dipole interaction enables strong coupling between the different $\left|J \Omega P M_{P}\right\rangle$ states, resulting in an inhomogeneous shift of the resonance positions and widths in the quantummechanical calculation as compared to the DIS approach. The dependence of the resonance positions and widths on the input potentials turns out to be rather straightforward. The existence of two bosonic isotopes of $\mathrm{Ne}^{*}$ enables us to choose the isotope with the most favorable scattering length for efficient evaporative cooling towards the Bose-Einstein condensation transition, greatly enhancing the feasibility to reach this transition.
\end{abstract}

DOI: 10.1103/PhysRevA.69.012706

PACS number(s): 34.50.-s, 34.20.Cf, 03.65.Nk, 03.75.Nt

\section{INTRODUCTION}

Bose-Einstein condensation (BEC) has been observed in cold dilute samples of ground-state alkali-metal atoms [1-4] and atomic hydrogen $[5,6]$. In 2001, the first condensate of atoms in an electronically excited state was obtained for metastable $\mathrm{He}\left[(1 s)(2 s)^{3} S_{1}\right][7,8]$, referred to as $\mathrm{He}^{*}$. All these systems have an electron configuration with only $s$ electrons in their open shells in common, resulting in an isotropic electrostatic interaction.

The other candidate for achieving BEC with atoms in an electronically excited state is metastable $\mathrm{Ne}\left[(2 p)^{5}(3 s)^{3} P_{2}\right]$, referred to as $\mathrm{Ne}^{*}$ in this paper. Two groups are pursuing this goal: the group of Ertmer in Hannover [9] and our group [10]. Metastable neon is unique among these species in that its binary electrostatic interaction is anisotropic, due to its $(2 p)^{-1}$ core hole [11].

Crucial in reaching the BEC phase transition is a large ratio of "good" to "bad" collisions, i.e., a large value of the elastic collision rate characterized by the total cross section $\sigma=8 \pi a^{2}$ for elastic collisions with $a$ the $s$-wave scattering length, and a small rate for inelastic collisions and other loss processes. In addition, the creation of a stable BEC requires a positive value of the scattering length. For metastable rare gas atoms, such as $\mathrm{He}^{*}$ and $\mathrm{Ne}^{*}$, the major loss process is Penning ionization in binary collisions. Fortunately, the latter process is suppressed in a sample of spin-polarized atoms [11]. For $\mathrm{He}^{*}$, the suppression is very efficient: only spin flips due to magnetic interactions result in some residual ionization. Theoretical predictions and recent experimental data on residual ionization are in good agreement [12-15].

For $\mathrm{Ne}^{*}$, the anisotropy in the electrostatic interaction determines the magnitude of the residual ionization in a spin-

*Email address: v.p.mogendorff@tue.nl polarized gas and has a profound influence on the value of the scattering length. Theoretical estimates of the rate constant $\beta^{p o l}$ for residual ionization of $\mathrm{Ne}^{*}$ predict a suppression of ionization by a factor in the range of $10-1000$ $[11,16]$, depending on the details of the interaction potentials. In experiments in Hannover and Eindhoven, a lower limit on the suppression of ionization by a factor of 10 has been confirmed, but so far no conclusive experimental data on the residual ionization rate of $\mathrm{Ne}^{*}$ are available.

In addition, the anisotropy in the interaction results in different interaction potentials $V_{\Omega}$ for the molecular states $|J, \Omega\rangle$ of the colliding $\mathrm{Ne}^{*}$ atoms, with $\Omega$ the absolute value of the projection of the total electronic angular momentum $\vec{J}=\vec{j}_{1}+\vec{j}_{2}$ of the two colliding atoms on the internuclear axis. For binary collisions of spin-polarized $\mathrm{Ne}^{*}$, we have $J=4$ and $\Omega=0$ through 4 , depending on the relative orientation of the atoms during the collision. This is illustrated in Fig. 1, which shows two colliding atoms in the $\Omega=0$ and $\Omega=4$ state, respectively, with the electronic angular momentum $j_{1,2}$ and the $(2 p)^{-1}$ orbital of the core hole indicated schematically.

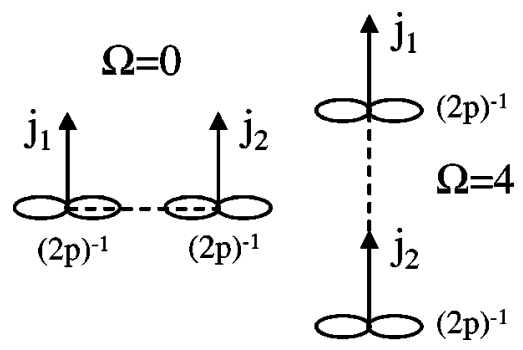

FIG. 1. Schematic view of two colliding $\mathrm{Ne}^{*}\left|{ }^{3} P_{2}\right\rangle$ atoms in a spin-polarized gas $(S=2, J=4)$ for both the $\Omega=0$ and the $\Omega=4$ state. The orientation of the electronic angular momentum $j_{1,2}$ and the $(2 p)^{-1}$ core hole of the individual atoms is indicated schematically. 


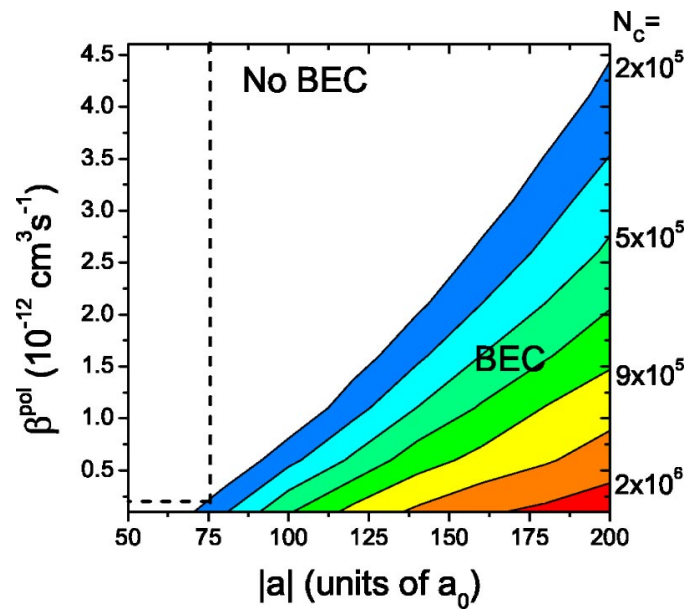

FIG. 2. Feasibility plot for reaching the BEC transition with ${ }^{20} \mathrm{Ne}^{*}$ in the Eindhoven experiment, showing the number of atoms $N_{c}$ with which quantum degeneracy is achieved as a function of the rate constant $\beta^{\text {pol }}$ for residual ionization and the absolute value of the scattering length $|a|$. The broken lines correspond to $a_{c}$ and $\beta_{c}^{p o l}$ for which BEC of $\mathrm{Ne}^{*}$ becomes feasible.

Because the scattering length is determined by the phase integral of the interaction potential, these different potentials $V_{\Omega}$ for $\mathrm{Ne}^{*}$ correspond to different scattering lengths $a_{\Omega}$. Since there is no preference for a certain relative orientation of the atoms (or $\Omega$ state) during the collision, even for spinpolarized $\mathrm{Ne}^{*}$, the elastic collision cross section will be determined by an effective overall scattering length $a$, incorporating the behavior of all five $\Omega$ states involved. Among the species where Bose-Einstein condensation has been achieved, the BEC candidate $\mathrm{Ne}^{*}$ thus has a unique property.

In this paper we investigate the relation between the effective overall scattering length $a$ and the phase integrals $\Phi_{\Omega}$ of the potentials $V_{\Omega}$. Although the potentials of $\mathrm{Ne}^{*}$ are unknown at the level of accuracy needed to predict the value of the scattering length, it is useful to investigate the behavior of the effective scattering length as a function of the average value of the phase integral. In the following, we will refer to the effective overall scattering length simply as scattering length.

A better understanding of the complex scattering length of $\mathrm{Ne}^{*}$ is crucial in determining the feasibility of achieving $\mathrm{BEC}$ with $\mathrm{Ne}^{*}$. Important questions that need to be answered to determine the feasibility for achieving BEC are the following.

(1) Is there a larger probability of encountering positive values of the scattering length, as compared to the $75 \%$ probability for the single-potential case?

(2) What is the probability for finding a sufficiently large elastic total cross section for efficient evaporative cooling?

(3) How does the availability of two bosonic isotopes of $\mathrm{Ne}^{*},{ }^{20} \mathrm{Ne}^{*}$ and ${ }^{22} \mathrm{Ne}^{*}$ (with a natural abundance of $90 \%$ and $10 \%$, respectively) influence these chances?

The values of the rate constant for residual ionization $\beta^{\text {pol }}$ and the scattering length $a$ which we need to achieve BEC with $\mathrm{Ne}^{*}$ for typical experimental conditions in our experiment, are summarized in Fig. 2. This so-called feasibility plot for achieving BEC with $\mathrm{Ne}^{*}$ shows the calculated number of atoms $N_{c}$ with which quantum degeneracy is achieved as a function of $\beta^{p o l}$ and the absolute value of the scattering length $|a|$. The calculation of this feasibility plot is based on the kinetic model of Luiten et al. [17], including trap losses. The criterion for crossing the BEC transition has been set to $N_{c}>1 \times 10^{5}$ atoms. The initial conditions of the evaporative cooling process are taken equal to experimental values which we are able to produce, i.e., $N=1.5 \times 10^{9}, T=1.2 \mathrm{mK}, \tau$ $=8 \mathrm{~s}$, and $\eta=5.5$, with $N$ the number of atoms, $T$ the temperature, $\tau$ the lifetime of the atom cloud, $\eta=E /\left(k_{B} T\right)$ the truncation parameter, $E$ the energy, and $k_{B}$ the Boltzmann constant.

From Fig. 2 it is clear that BEC of $\mathrm{Ne}^{*}$ is feasible for $\beta_{c}^{p o l} \leqslant 2.5 \times 10^{-13} \mathrm{~cm}^{3} \mathrm{~s}^{-1}$ (a suppression of 500) and $a_{c}$ $\geqslant 75 a_{0}$, with $a_{0}$ the Bohr radius. Therefore, we calculate the probability $P_{c}$ that either of the bosonic isotopes of $\mathrm{Ne}^{*}$ has a total cross section larger than $\sigma_{c}=8 \pi a_{c}^{2}=\left(1.4 \times 10^{5}\right) a_{0}^{2}$. A larger suppression of ionization of course allows for a smaller value of the scattering length and vice versa.

As a first-order estimate, in a simple gas-kinetic picture, one might expect the effective elastic total cross section $\sigma$, referred to as elastic cross section in the remainder of this paper, to be a weighted average over the elastic cross sections $\sigma_{\Omega}=8 \pi a_{\Omega}^{2}$ of the different molecular states. Applying this approach to the anisotropic $\mathrm{Ne}^{*}$ problem results in a large enhancement of the probability of encountering large values of $\sigma$ as compared to all systems with an isotropic interaction potential. Moreover, the elastic cross section is always larger than a rather large lower limit.

A more sophisticated approach can be found in the degenerate internal state (DIS) method [23]. In this method, the energy splitting of the internal states is neglected and $a$ is given by the weighted average of the contributing scattering lengths $a_{\Omega}$. For cold collisions of hydrogen atoms, the DIS method results in values for the scattering length that compare well to the outcome of full quantum calculations $[18,19]$.

The definitive approach to determine the scattering length $a$ of $\mathrm{Ne}^{*}$, of course, is a full five-channel quantummechanical calculation. Although this numerical approach supplies the correct answer to our problem, it has the disadvantage that the results are not always easy to understand in terms of the properties of the input potentials. We use the results of the numerical calculation to check the validity of the different analytical approximations described above, which in general give more insight.

This paper is organized as follows. First, the available $a b$ initio potentials and the calculation of the different phase integrals $\Phi_{\Omega}$ are discussed in Sec. II. In Sec. III the singlepotential scattering length (Sec. III A), the gas-kinetic approach to defining an elastic cross section $\sigma$ (Sec. III B), and the scattering length obtained with the DIS approximation (Sec. III C) are discussed. We then present the results of our quantum-mechanical, numerical scattering calculation in Sec. IV. To end we present our conclusions in Sec. V. All calculations are performed for both bosonic isotopes of $\mathrm{Ne}^{*}$, ${ }^{20} \mathrm{Ne}^{*}$, and ${ }^{22} \mathrm{Ne}^{*}$. 


\section{INTERACTION POTENTIALS}

There are five adiabatic molecular states $|J, \Omega\rangle$ that connect to the spin-polarized $\mathrm{Ne}^{*}+\mathrm{Ne}^{*}$ asymptotic limit with total electronic angular momentum $J=4$ and total spin $S=2$. The degeneracy of the $\Omega=0$ state is 1 , that of all others is equal to 2 . As input potentials for $\mathrm{Ne}^{*}$ we have used the short-range $a b$ initio potentials of Kotochigova et al. [20,21], which are available in the range $R \leqslant R_{1}$, with $R_{1}=60 a_{0}$ for $\Omega=4$ and $R_{1}=120 a_{0}$ for all other potentials. Typical values of the well depth $\epsilon$ and its position $R_{m}$ are $\epsilon \approx 30 \mathrm{meV}$ and $R_{m} \approx 10 a_{0}$. The long-range behavior of the potential curves is dominated by the attractive induced dipole-dipole interaction $-C_{6} / R^{6}$. The $a b$ initio potentials have within $\leqslant 3 \%$ identical $C_{6}$ coefficients, since the longrange interaction is dominated by the $(3 s)$ valence electron $[11,16]$. We use a single $C_{6}$ coefficient with a value of $C_{6}=1938$ a.u. as calculated by Derevianko and Dalgarno [16], based on the static polarizability of neon.

We characterize the potentials $V_{\Omega}$ by their classical phase integral

$$
\Phi_{\Omega}=\int_{R_{c}}^{R_{s}} k_{\Omega}(R) d R+\int_{R_{s}}^{\infty} k_{\Omega}(R) d R=\Phi_{\Omega}^{R<R_{s}}+\Phi^{R>R_{s}},
$$

with $k_{\Omega}(R)$ the local wave number and $R_{c}$ the classical inner turning point for zero collision energy. We choose $R_{S}$ such that for $R<R_{s}$ the energy splitting $\Delta V_{\Omega, \Omega^{\prime}}(R)=V_{\Omega}(R)$ $-V_{\Omega^{\prime}}(R)$ between the $\Omega$ potentials dominates over the rotational coupling

$$
\begin{aligned}
\Delta V_{\text {rot }}(R)= & -\left[\hbar^{2} /\left(2 \mu R^{2}\right)\right] \delta_{\left|\Omega-\Omega^{\prime}\right|=1} \\
& \times \sqrt{\left[P(P+1)-\Omega \Omega^{\prime}\right]} \sqrt{\left[J(J+1)-\Omega \Omega^{\prime}\right]},
\end{aligned}
$$

with $\mu$ the reduced mass, $l$ the rotational angular momentum, and $\vec{P}=\vec{J}+\vec{l}$ the total angular momentum. For $R>R_{s}$ the opposite holds.

The first part $\Phi_{\Omega}^{R<R_{s}}$ of the phase integral has been calculated by numerical integration in the interval $\left[R_{c}, R_{s}\right]$, with $R_{s}=20 a_{0}$. The contribution for $R>R_{s}$ to the phase integral is calculated analytically assuming a pure long-range, $-C_{6} / R^{6}$, behavior, resulting in $\Phi^{R>R_{s}}=3.34 \pi$.

The numerical results are given in Table I for both bosonic isotopes, ${ }^{20} \mathrm{Ne}^{*}$ and ${ }^{22} \mathrm{Ne}^{*}$. We see that the different $\Omega$ states have very different values of $\Phi_{\Omega}$, varying by as much as $\Delta \Phi_{\Omega, \Omega^{\prime}}=\Phi_{\Omega}-\Phi_{\Omega^{\prime}}=0.8 \pi$. This implies both a different number of bound states and different positions of the resonances in $a_{\Omega}$. Asymptotic behavior of, or a resonance in the scattering length occurs when a quasibound state lies close to the dissociation limit or has just moved into the continuum.

Because the interaction potentials of neon are not known accurately enough to predict $a$, we have to vary $\Phi_{\Omega}$ over a range equal to $\pi$ to predict the range of $a$ values that we can expect for the spin-polarized $\mathrm{Ne}^{*}$ system. Therefore, we introduce a scanning parameter $\phi$ that we add to $\Phi_{\Omega}$ to create a modified phase integral $\phi_{\Omega}$ according to
TABLE I. Classical phase integral $\Phi_{\Omega}$ and its difference $\Delta \Phi_{\Omega, 0}=\Phi_{\Omega}-\Phi_{0}$ with respect to the $\Omega=0$ potential of the spinpolarized adiabatic molecular $\Omega$ states, connecting to the $\mathrm{Ne}^{*}$ $+\mathrm{Ne}^{*}$ asymptotic limit with $J=4$ and $S=2$. The $\Omega$ states are labeled by $\Omega_{g}$, where the gerade label $g$ reflects the symmetry of the electron wave function under inversion around the center of charge. Data are given for both bosonic isotopes ${ }^{20} \mathrm{Ne}^{*}$ and ${ }^{22} \mathrm{Ne}^{*}$ of $\mathrm{Ne}^{*}$.

\begin{tabular}{lcccc}
\hline \hline & \multicolumn{2}{c}{${ }^{20} \mathrm{Ne}^{*}$} & \multicolumn{2}{c}{${ }^{22} \mathrm{Ne}^{*}$} \\
$\Omega$ & $(\pi \mathrm{rad})$ & $\begin{array}{c}\Delta \Phi_{\Omega, 0} \\
(\pi \mathrm{rad})\end{array}$ & $\begin{array}{c}\Phi_{\Omega} \\
(\pi \mathrm{rad})\end{array}$ & $\begin{array}{c}\Delta \Phi_{\Omega, 0} \\
(\pi \mathrm{rad})\end{array}$ \\
\hline $4_{g}$ & 16.43 & -0.54 & 17.23 & -0.77 \\
$3_{g}$ & 16.86 & -0.11 & 17.68 & -0.34 \\
$2_{g}$ & 16.12 & -0.85 & 16.91 & -1.09 \\
$1_{g}$ & 16.36 & -0.61 & 17.16 & -0.84 \\
$0_{g}$ & 16.97 & 0 & 18.00 & 0 \\
\hline \hline
\end{tabular}

$$
\phi_{\Omega}=\Phi_{\Omega}+\phi
$$

with $\phi \in[0, \pi]$.

For simplicity, we assume for now that the phase differences between the $\Omega$ potentials $\Delta \Phi_{\Omega, \Omega^{\prime}}$ are constant and equal to the $a b$ initio values given in Table I. Later on in Sec. IV, we will also vary $\Delta \Phi_{\Omega, \Omega^{\prime}}$ over an interval $\pi$ to investigate the influence of $\Delta \Phi_{\Omega, \Omega^{\prime}}$ on the scattering length $a$.

The classical phase integrals of the two bosonic isotopes of $\mathrm{Ne}^{*}$ are related by the mass-scaling rule

$$
{ }^{22} \phi_{\Omega}=(22 / 20)^{1 / 220} \phi_{\Omega} .
$$

Using the average phase integral over all $\Omega$ states $\langle\Phi\rangle_{\Omega}$ $=16.5 \pi$, we find an isotope shift equal to $0.81 \pi$. This simple relation between the phase integrals of ${ }^{20} \mathrm{Ne}^{*}$ and ${ }^{22} \mathrm{Ne}^{*}$ enables us to compare very easily the results obtained for ${ }^{20} \mathrm{Ne}^{*}$ with those for ${ }^{22} \mathrm{Ne}^{*}$.

\section{ANALYTICAL APPROACH}

\section{A. Single-potential scattering length}

The semiclassical analysis of the scattering length in atomic collisions by Gribakin and Flambaum [22] yields for the $s$-wave scattering length of a potential with a long-range behavior, $-C_{6} / R^{6}$,

$$
\begin{gathered}
a_{\Omega}=a_{b g}\left[1-\tan \left(\phi_{\Omega}-\frac{\pi}{8}\right)\right], \\
a_{b g}=\cos (\pi / 4)\left[\sqrt{2 \mu C_{6}} / 4 \hbar\right]^{1 / 2}[\Gamma(3 / 4) / \Gamma(5 / 4)],
\end{gathered}
$$

with $\Gamma\left(\right.$ ) the Gamma function and $a_{b g}$ the background value of the scattering length. The latter is fully determined by the long-range behavior of the potential and is equal to $a_{b g}$ $=44.3 a_{0}$. The position of the resonance in $a_{\Omega}$ is equal to $\phi_{\Omega}^{r e s} \bmod \pi=\pi / 2+\pi / 8=5 \pi / 8$. We define the width $\Gamma_{\Omega}$ of a resonance in $a_{\Omega}$ as

$$
\Gamma_{\Omega}=\phi_{\Omega}\left(3 a_{b g}\right)-\phi_{\Omega}\left(-a_{b g}\right)
$$




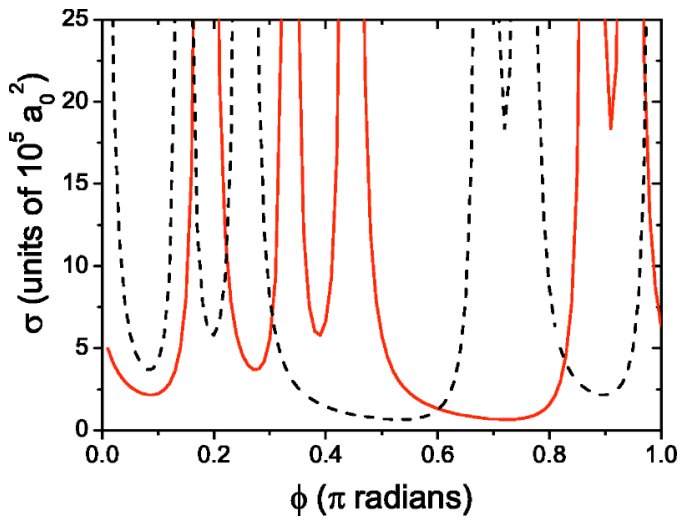

FIG. 3. Elastic cross section $\sigma=\left\langle 8 \pi a_{\Omega}^{2}\right\rangle$ in a gas-kinetic approach for ${ }^{20} \mathrm{Ne}^{*}$ (solid line) and ${ }^{22} \mathrm{Ne}^{*}$ (broken line), as a function of the phase integral $\phi \in[0, \pi]$. We observe five resonances due to the five contributing potentials, which also results in a minimum value $\sigma_{\min }>0$ for $\sigma$.

around the resonance position $\phi_{\Omega}^{\text {res }}$. The scattering length varies around $a_{b g}$ with a probability of $75 \%$ of being positive. The probability that at least one of the bosonic isotopes of $\mathrm{Ne}^{*}$ is positive is much larger, $94 \%$.

\section{B. Gas-kinetic model}

In a simple gas-kinetic approach, we define the elastic cross section as a weighted average of the elastic cross sections $\sigma_{\Omega}$,

$$
\sigma=\left\langle 8 \pi a_{\Omega}^{2}\right\rangle=\sigma_{b g} \sum_{\Omega=0}^{J} w_{\Omega}^{2}\left[1-\tan \left(\phi_{\Omega}-\frac{\pi}{8}\right)\right]^{2} .
$$

Here $\sigma_{b g}=8 \pi a_{b g}^{2}=\left(0.5 \times 10^{5}\right) a_{0}^{2}$ is the background value of the elastic cross section and $w_{\Omega}$ is the amplitude of the projection of the initial asymptotic rotational state on the $\Omega$ basis, with $w_{0}=1 / 3$ and $w_{\Omega>0}=\sqrt{2} / 3$. We assume that we are in the low-temperature limit where the condition $k a \ll 1$ holds, with $k=\sqrt{2 \mu E / \hbar}$ the asymptotic wave number and $E$ the collision energy in the reduced system.

In Fig. 3 we show the results for the elastic cross section of ${ }^{20} \mathrm{Ne}^{*}$ (solid line) and ${ }^{22} \mathrm{Ne}^{*}$ (broken line) as a function of $\phi \in[0, \pi]$ [Eq. (2)]. Two important characteristics in the elastic cross section of $\mathrm{Ne}^{*}$ immediately catch the eye. First, we see a rather large minimum value $\sigma_{\min }$ for the elastic cross section and an increase of almost a factor of 2 in the probability $P_{c}$ that the elastic cross section is large enough to make $\mathrm{BEC}$ of $\mathrm{Ne}^{*}$ feasible as compared to the singlepotential case, as can be seen in Table II.

Second, we see five resonances in $\sigma$, attributable to the five different $\Omega$ states of $\mathrm{Ne}^{*}$. This characteristic behavior does not depend very much on the specific values of $\Delta \Phi_{\Omega, 0}$ as long as they are not very small $(\leqslant 0.05 \pi)$. This behavior is very different from the single-potential case, where we can encounter an elastic cross section equal to zero and there exists only one resonance.

The general picture is the same for both isotopes. However, the elastic cross section of ${ }^{22} \mathrm{Ne}^{*}$ is shifted with respect
TABLE II. Minimum value $\sigma_{\min }$ of the elastic cross section and probability $P_{c}$ for $\sigma \geqslant \sigma_{c}=\left(1.4 \times 10^{5}\right) a_{0}^{2}$ for a single isotope $\left({ }^{20} \mathrm{Ne}^{*}\right)$ and the set of two bosonic isotopes ${ }^{20} \mathrm{Ne}^{*}$ and ${ }^{22} \mathrm{Ne}^{*}$, as calculated with the single-potential semiclassical model, the gaskinetic model, the DIS model, and the quantum-mechanical numerical calculation.

\begin{tabular}{ccccc}
\hline \hline & \multicolumn{2}{c}{ Single isotope } & \multicolumn{2}{c}{ Either isotope } \\
Model & $\begin{array}{c}\sigma_{\min } \\
\left.\text { (units of } 10^{5} a_{0}^{2}\right)\end{array}$ & $\begin{array}{c}P_{c} \\
(\%)\end{array}$ & $\begin{array}{c}\sigma_{\min } \\
\left.\text { (units of } 10^{5} a_{0}^{2}\right)\end{array}$ & $\begin{array}{c}P_{c} \\
(\%)\end{array}$ \\
\hline Single potential & 0 & 42 & 0.16 & 61 \\
Gas kinetic & 0.7 & 78 & 1.3 & 99 \\
DIS & 0 & 72 & 0.35 & 95 \\
Numerical & 0 & 42 & 0.17 & 67 \\
\hline \hline
\end{tabular}

to the elastic cross section of ${ }^{20} \mathrm{Ne}^{*}$ by the isotope shift of $0.81 \pi$. Depending on the actual phase integral $\phi_{\Omega}$ of the ${ }^{20} \mathrm{Ne}^{*}$ system, it can thus be advantageous to switch to the less abundant bosonic isotope ${ }^{22} \mathrm{Ne}^{*}$, to optimize the value of the elastic cross section. Choosing for each value of $\phi$ the isotope with the largest $\sigma$ yields an even larger minimum value of $\sigma$, as can be seen in Table II. In addition, $P_{c}$ increases from $78 \%$ in the single-isotope case to $99 \%$ for either isotope.

\section{DIS model}

Next, we investigate the scattering length in the DIS approximation, which has proven to be quite insightful for hydrogen $[18,19]$. In this approach, the energy splitting of the internal states is neglected. For $\mathrm{Ne}^{*}$, this means that the rotational splitting $\Delta V_{\text {rot }}$ between the partial waves is neglected. In the DIS approximation, the scattering length is given by a weighted average of the five $a_{\Omega}$ 's involved [19]

$$
a=\left\langle a_{\Omega}\right\rangle=a_{b g} \sum_{\Omega=0}^{4} w_{\Omega}\left[1-\tan \left(\phi_{\Omega}-\frac{\pi}{8}\right)\right] .
$$

From Eq. (7) it is clear that the resonance positions $\phi_{\Omega}^{\text {res }}$ of $a$ in the DIS approximation, coincide with the single-potential resonance positions. They are completely determined by the values of $\Delta \Phi_{\Omega, 0}$,

$$
\phi_{\Omega}^{r e s}=\left(\frac{5}{8} \pi-\Delta \Phi_{\Omega, 0}\right) \bmod \pi .
$$

Figure 4 shows the scattering length $a$ as a function of the scanning parameter $\phi \in[0, \pi]$. Again we observe five resonances in the scattering length. In this figure we have also plotted the behavior of the single-potential scattering length $a_{\Omega=4}$ (broken line), showing clearly that the single-potential resonance positions coincide with the resonance positions of $a$ in the DIS approach.

Taking the weighted average of $a_{\Omega}$ does not change the total probability for a positive scattering length $(75 \%)$ as compared to the single-potential case (Sec. III A). However, the probability of encountering a large value of $a$ does increase, as can be seen in Table II. Both the width of the 


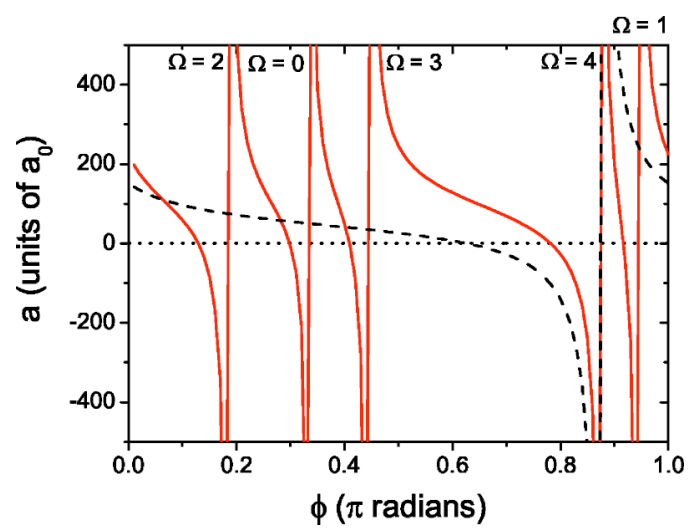

FIG. 4. DIS result for the scattering length $a$ (solid line) as a function of the parameter $\phi \in[0, \pi]$, showing five resonances that are labeled with an $\Omega$ value because they are located at the position of the single-potential resonances in $a_{\Omega}$. For comparison we show the behavior of $a_{4}$ (broken line).

resonances [Eq. (5)] and the derivative $\partial a / \partial \phi$ of $a$ with respect to $\phi$ determine this probability. From Fig. 4 it is clear that the total width $\Gamma$ of all five resonances combined is much larger than the single-potential resonance width. The width of each resonance is not only determined by the relative weight $w_{\Omega}$ of its single-potential scattering length but also by its relative position $\phi_{\Omega}^{\text {res }}$ with respect to the other resonances, and therefore depends sensitively on the phase differences $\Delta \Phi_{\Omega, \Omega^{\prime}}$ between the potentials. An increased width and derivative of $a$ in the DIS approach as compared to the single-potential case therefore lead to a much larger $P_{c}$ (Table II). Choosing the most advantageous bosonic isotope improves again $\sigma_{\min }$ and $P_{c}$ as well as the probability for encountering a positive value of $a(95 \%)$ as compared to the single-isotope case (Table II).

\section{QUANTUM-MECHANICAL NUMERICAL CALCULATION}

\section{A. Model}

For the $\mathrm{Ne}^{*}$ system, we can distinguish two regions of interest in the potentials $V_{\Omega}(R)$ (Fig. 5). At small internu-

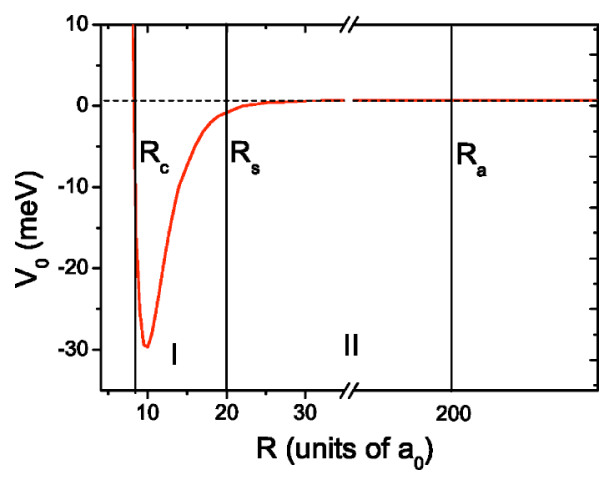

FIG. 5. Interaction potential $V_{0}$ for the $\Omega=0$ molecular state of two colliding spin-polarized $\mathrm{Ne}^{*}$ atoms, with both range I (dominant $\Omega$ splitting) for $R \leqslant R_{s}=20 a_{0}$ and range II (dominant rotational energy splitting) for $R_{s}<R<R_{a}=200 a_{0}$ indicated. clear separations (region I), i.e., $R<R_{s}$, the splitting $\Delta V_{\Omega, \Omega^{\prime}}(R)$ between the interaction potentials $V_{\Omega}$ is larger than the differential rotational coupling $\Delta V_{r o t}(R)$. As a result, there is no coupling between different $\Omega$ states but coupling between different $l$ states. In this region $\Omega$ is a good quantum number and the $\left|J \Omega P M_{P}\right\rangle$ basis is the proper representation. Here $M_{P}$ is the projection of $P$ on the quantization axis. Both $P$ and $M_{P}$ are conserved during the collision. In a semiclassical picture we can describe this regime in the following way. The large $\Omega$-splitting results in a rapid precession of $\vec{J}$ about the internuclear axis, much faster than the rotation of the axis itself as determined by the value of $|\vec{l}|$. The projection of $\vec{J}$ on the internuclear axis is thus conserved, while the magnitude of $\vec{l}$ changes due to the changing orientation of $\vec{J}$ with respect to the space-fixed total angular-momentum vector $\vec{P}$.

At large internuclear separations (region II in Fig. 5), i.e., $R>R_{s}$, the rotational coupling dominates the interaction: the splitting $\Delta V_{\Omega, \Omega^{\prime}}(R)$ is smaller than the differential rotational coupling $V_{\text {rot }}(R)$. The relative motion of the colliding atoms results in a rotation of the internuclear axis with respect to $\vec{J}$ and therefore a change in the value of $\Omega$ (Fig. 1). In this region $\Omega$ is not a good quantum number but $l$ is, and we use the $\left|J l P M_{P}\right\rangle$ representation.

Ultracold collisions between spin-polarized $\mathrm{Ne}^{*}$ atoms are described by a five-channel problem: five $\Omega=0, \ldots, 4$ channels in region I and five $l=0,2, \ldots, 8$ channels in region II. Only even partial waves contribute due to the symmetry requirement of the wave function for bosons. The rotational energy barrier $\left(5.6 \mathrm{mK}\right.$ for $l=2$, located at $\left.78 a_{0}\right)$ is always much larger than the collision energy $(\leqslant 0.5 \mathrm{mK})$. For this reason, higher-order partial waves $(l \neq 0)$ do not contribute to the incoming channel. However, in region I, the shortrange interaction $\Delta V_{\Omega, \Omega^{\prime}}$ still couples the single incoming channel $\left|J=4 \quad l=0 \quad P=4 \quad M_{P}=4\right\rangle$ to higher-order partial waves. Because the tunneling probability for $l \neq 0$ is negligible $\left(\leqslant 10^{-5}\right)$, they only contribute to the elastic scattering process when they again couple to the $\mid J=4 \quad l=0 \quad P=4$ $\left.M_{P}=4\right\rangle$ initial state.

In our calculations we assume that the intermediate region, where $V_{\text {rot }}(R)$ and $V_{\Omega, \Omega^{\prime}}(R)$ are of the same order of magnitude, is arbitrarily small, i.e., we assume a sudden transition from region I to region II at $R=R_{s}$. The scattering problem now reduces to potential scattering and we can solve the uncoupled problem in region I in the $\left|J \Omega P M_{P}\right\rangle$ basis and in region II in the $\left|J l P M_{P}\right\rangle$ basis, for each channel. After transformation of the solution $u_{\mathrm{I}}(R)$ in region $\mathrm{I}$ at $R$ $=R_{S}$ from the $\left|J \Omega P M_{P}\right\rangle$ basis to the $\left|J l P M_{P}\right\rangle$ basis, we connect it continuously to the long-range solution $u_{\mathrm{II}}(R)$ at $R_{s}$, assuming equal local wave numbers [23].

In very good approximation $[24,25]$, we can summarize the behavior of the atoms in region I by means of the accumulated phase method. In $R=R_{s}$ the radial wave function of a single uncoupled channel is then given by

$$
u_{\mathrm{I}}^{\Omega}\left(R_{s}\right)=\sin \left(\Phi_{\Omega}^{R<R_{s}}+\phi+\pi / 4\right),
$$




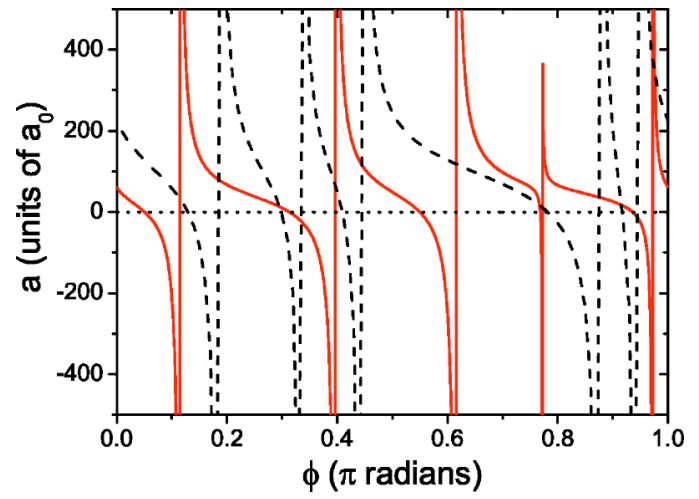

FIG. 6. Full quantum-mechanical calculation of the scattering length $a$ (solid line) as a function of $\phi \in[0, \pi]$. For comparison we also have depicted the scattering length $a$ obtained with the DIS model (broken line). Both position and width of the resonances in $a$ differ from the DIS result: an $\Omega$ label cannot be attached to any separate resonance.

with the extra phase shift $\pi / 4$ due to our choice of using the classical phase rather than the quantum-mechanical accumulated phase. After transformation to the $\left|J l P M_{P}\right\rangle$ basis the $5 \times 5$ solution matrix is given by

$$
u_{\mathrm{I}}^{l}\left(R_{s}\right)=T_{l \Omega} u_{\mathrm{I}}^{\Omega}\left(R_{s}\right),
$$

with $T_{l \Omega}$ the elements of the transformation matrix $\underline{\mathbf{T}}$ between the $\left|J \Omega P M_{P}\right\rangle$ and the $\left|J l P M_{P}\right\rangle$ basis.

In region II, the evolution of the radial wave function $u_{\mathrm{II}}^{l}$ for $R>R_{s}$ is governed by the radial Schrödinger equation

$$
\frac{\partial^{2}}{\partial R^{2}} u_{\mathrm{II}}^{l}(R)+\left(k^{2}-\frac{l(l+1)}{R^{2}}-\frac{2 \mu C_{6}}{\hbar^{2} R^{6}}\right) u_{\mathrm{II}}^{l}(R)=0 .
$$

The connection of the short-(I) and long-range (II) solutions in the $\left|J l P M_{P}\right\rangle$ representation at $R=R_{S}$ determines the boundary conditions for the numerical integration of $u_{\mathrm{II}}^{l}$ from $R_{s}$ to $R_{a}$, where the asymptotic limit of a vanishing potential is valid. At $R_{a}$, the numerical solution is connected to the asymptotic radial wave function

$$
\left.u_{\mathrm{II}}^{l}\left(R_{a}\right)=\frac{1}{\sqrt{k}}\left[A_{l} e^{-i\left(k R_{a}-l \pi / 2\right)}+B_{l} e^{i\left(k R_{a}-l \pi / 2\right)}\right)\right],
$$

to determine the scattering matrix $\underline{\mathbf{S}}=\underline{\mathbf{B A}}^{-1}$. From the scattering matrix we then obtain the scattering length $a$,

$$
a=-\lim _{k \rightarrow 0} \frac{\tan \left[\ln \left(S_{00}\right) / 2 i\right]}{k} .
$$

\section{B. Results}

Using the $a b$ initio potentials of Sec. II we have calculated the scattering length of ${ }^{20} \mathrm{Ne}^{*}$ using the quantummechanical calculation described in Sec. IV A. Again, we have performed these calculations for $\phi \in[0, \pi]$, to determine the range of scattering length and elastic cross section values that we can expect for the $\mathrm{Ne}^{*}$ system. Figure 6 shows the scattering length $a$ (solid line) as a function of $\phi$. For comparison, we have included the result for $a$ obtained with the DIS model (broken line) (Sec. III C), in which the positions of the resonances in $a$ correspond to the singlepotential resonance positions in $a_{\Omega}$. The resonance positions $\phi_{\Omega}^{\text {res }}$ of $a$ are shifted in an inhomogeneous way with respect to those obtained with the DIS method and also the widths $\Gamma_{\Omega}$ of the individual resonances differ significantly. We can no longer attach an $\Omega$ label to each of the resonances, as is possible in the DIS approach. In the DIS model, we neglect the rotational splitting $V_{\text {rot }}$ between the partial waves, so that effectively all partial waves are equivalent. Apparently, both the resonance positions as well as the widths of the individual resonances in the numerical results for $a$ are influenced by the coupling to higher-order partial waves. This is not surprising: close lying bound states from other $\Omega$ potentials will most likely shift the bound state and thus the corresponding resonance position. Clearly, neglecting the rotational splitting of the internal states is not justified in the case of $\mathrm{Ne}^{*}$.

However, despite these qualitative differences in the behavior of $a$, the quantitative behavior is the same as in the single-potential case. The probabilities for encountering a positive $a$ value and an elastic cross section $\sigma_{c}$ are equal to those found in the single-potential case (Table II). Although the total width of the resonances is larger, this is compensated by a decrease in the derivative of $a$. Moreover, choosing the most advantageous bosonic isotope for each value of the scanning parameter $\phi$ leads to a similar increase in these values for both the full quantum-mechanical calculation as the single-potential case.

To investigate the influence of $\Delta \Phi_{\Omega, \Omega^{\prime}}$ on the positions of the resonances in $a$, we have varied the classical phase difference $\Delta \Phi_{4,0}$ of one of the $\Omega$ potentials $(\Omega=4)$, while keeping the other classical phase differences fixed at their $a b$ initio value (Table I). Starting at its ab initio value, the classical phase difference

$$
\Delta \phi_{4,0}=\Delta \Phi_{4,0}+\Delta \phi
$$

with $\Delta \phi \in[0, \pi]$, is varied over $\Delta \phi_{4,0} \in[-0.54 \pi, 0.46 \pi]$. In this way, the bound states in the $\Omega=4$ potential encounter the bound states in all other $\Omega$ potentials. The position of the resonances $\phi_{\Omega}^{\text {res }}$ for this modified set of ab initio potentials is determined in the usual way, by scanning the parameter $\phi$ in the phase integral $\phi_{\Omega}$ [Eq. (2)] over the range $[0, \pi]$.

The results are shown in Fig. 7, where we have plotted the position $\phi_{\Omega}^{\text {res }}$ of the five resonances in $a$ as a function of the shift $\Delta \phi$ in the bound states of the $\Omega=4$ potential. The broken lines are drawn to guide the eye. We observe that two of the resonances remain at a fixed position, while all three others shift proportional to $\Delta \phi$ with a slope equal to $(-0.34 \pm 0.02) \pi$ and separated by $(0.30 \pm 0.02) \pi$. Narrow avoided crossings occur when "constant" $\phi_{\Omega}^{\text {res }}$ meet $\phi_{\Omega}^{\text {res }}$ varying like $\propto-\Delta \phi$ at $\Delta \phi=(0.46+n) \pi$ and $\Delta \phi=(0.52$ $+n) \pi$, with $n=0,1,2, \ldots$. In addition, very broad avoided crossings occur between $\phi_{\Omega}^{\text {res }}$ varying like $\propto-\Delta \phi$ at $\Delta \phi$ $\approx(0.1+n) \pi$, with $n=0,1,2, \ldots$. We have plotted $\phi_{\Omega}^{\text {res }}$ 


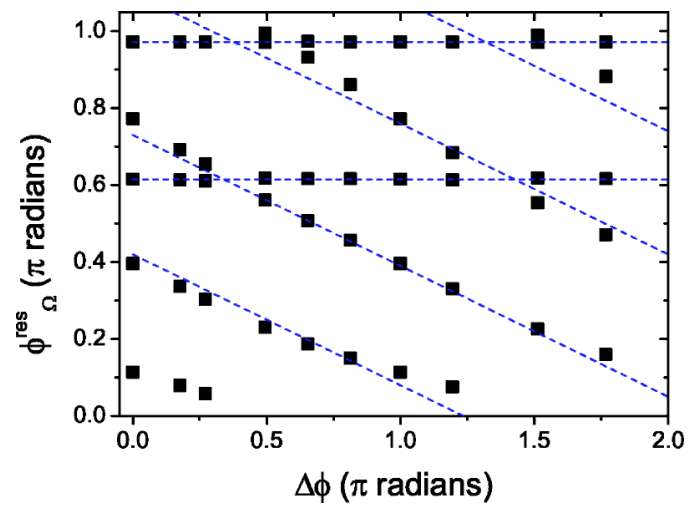

FIG. 7. Numerical calculation of the phase integrals $\phi_{\Omega}^{\text {res }}$ (filled squares) at which a resonance in $a$ occurs for a modified set of potentials, with the phase integral difference $\Delta \phi_{4,0}$ varying from its ab initio value $-0.54 \pi$ to $+1.5 \pi$ by varying $\Delta \phi$ over $2 \pi$. In this way, the bound levels of the $\Omega=4$ state "encounter" the bound levels in all other $\Omega$ states. The broken lines are drawn to guide the eye.

over a range $\Delta \phi \in[0,2 \pi]$, to show the avoided crossings. Varying one of the other classical phase differences $\Delta \phi_{\Omega \neq 4,0}$ yields similar results.

Clearly, the quasibound states of the system are no longer pure $\left|J \Omega P M_{P}\right\rangle$ states. Apparently, the quasibound states whose resonance positions $\phi_{\Omega}^{\text {res }}$ vary proportional to $\Delta \phi$ are strongly coupled to the $\left|J \Omega=4 P M_{P}\right\rangle$ state and those whose $\phi_{\Omega}^{\text {res }}$ remain constant are only very weakly coupled to the $\left|J \Omega=4 P M_{P}\right\rangle$ state. When two strongly coupled resonances approach each other, a strong, broad avoided crossing occurs. Similarly, a weak, narrow avoided crossing occurs when a weakly coupled resonance approaches one of the other resonances.

This picture is consistent with the behavior of the width of the resonances as a function of $\Delta \phi$. This becomes clear when we look at a simpler, more transparent two-channel, i.e., $J=1$, numerical calculation of the scattering length. The results of this calculation are consistent with the full fivechannel calculation. In Fig. 8(a) we have plotted the phase integrals $\phi_{\Omega}^{\text {res }}$ at which a resonance in $a$ occurs as a function of $\Delta \phi$ for $J=1$. Both resonance positions vary proportional to $\Delta \phi$ with a slope equal to $(-0.50 \pm 0.02) \pi$, and with broad avoided crossing between them at $\Delta \phi=(0.1+n) \pi$, with $n=0,1, \ldots$. They are strongly coupled, which is also reflected in the behavior of the width of the resonances $\Gamma_{\Omega}$ [Fig. 8(c)], which varies as a sine (broken line) between 0 and $\Gamma$. At the avoided crossings the widths of the resonances become equal. The total width of both resonances combined $\Gamma=\Gamma_{0}+\Gamma_{1}$ is conserved, but one resonance is wide while the other is narrow. This periodic change in the resonance width is due to the periodic change in the coupling of both quasibound states to the incoming $l=0$ channel with changing $\phi_{\Omega}^{\text {res }}$.

The weakly coupled case can be illustrated by reducing the value of $C_{6}$, because in the absence of an induced dipoledipole interaction $-C_{6} / R^{6}$ no coupling between the quasibound states is possible. This can be understood in the following way. With a decreasing dipole-dipole interaction the
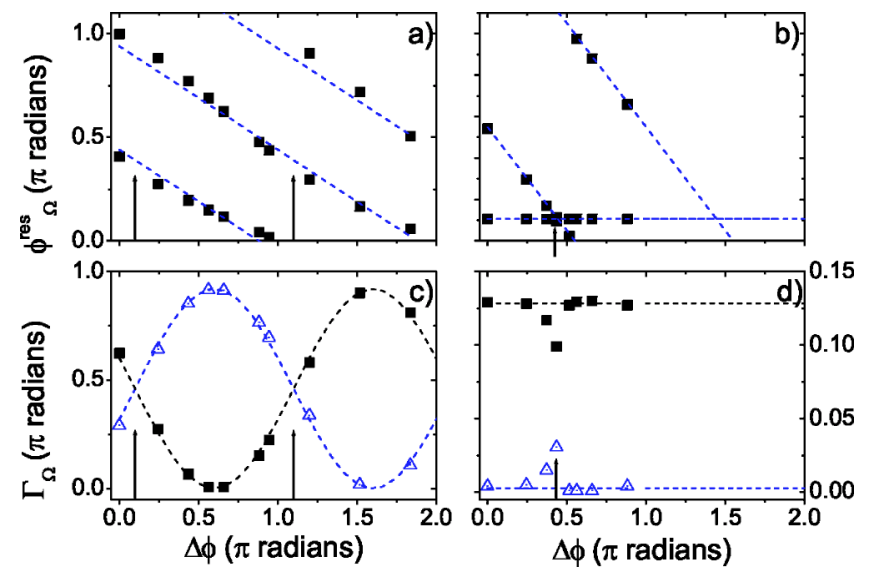

FIG. 8. Numerical two-channel $(J=1)$ calculation of position (a) and (b) and width (c) and (d) of the resonances in $a$ for a modified set of potentials, with $\Delta \phi_{1,0}$ varying from its ab initio value $-0.61 \pi$ to $+1.39 \pi$ by varying $\Delta \phi$ over $2 \pi$. The figures on the right-hand side (b) and (d) show the results for a reduced $C_{6}^{\prime}$ $=C_{6} / 100$. The broken lines are drawn to guide the eye and the arrows indicate the avoided crossings. In the presence of an induced dipole-dipole interaction (a) the resonance positions vary both proportional to $\Delta \phi$ and are strongly coupled, and the width of the resonances (c) varies as a sine. For a small dipole-dipole interaction (b) one of the resonance positions remains constant, while the other varies proportional to $\Delta \phi$. The width of the resonances (d) remains constant, except at the avoided crossing where they coincide (arrow).

rotational energy barrier increases both in height and width. As a result, the region around $R_{s}$ in which rotational coupling between the different $\Omega$ states takes place decreases and eventually vanishes. Figures 8(b) and 8(d) show the resonance positions and widths for a vanishing value of $C_{6}$, simulated by assuming a modified value $C_{6}^{\prime}=C_{6} / 100$. One resonance position remains constant, while the other varies proportional to $\Delta \phi$ and the widths of the resonances are small and remain constant, except at the avoided crossing where they approach each other. This is the behavior seen for some of the resonances in $a$ for $J=4$ and is qualitatively the same as the behavior of the resonance positions and widths in the DIS model, where coupling between the quasibound states is not taken into account.

\section{CONCLUDING REMARKS}

Elastic collisions between spin-polarized $\mathrm{Ne}^{*}$ atoms are governed by multiple interaction potentials. This unique property of $\mathrm{Ne}^{*}$ among the BEC species and candidates is a result of the anisotropic interaction between them. Both simple analytical and full quantum-mechanical calculations of the scattering length $a$ of $\mathrm{Ne}^{*}$ show that the resulting scattering length has five resonances. A simple gas-kinetic picture yields very favorable but unrealistic results for the elastic collision cross section that are not compatible with the numerical calculations. This approach is only valid for an incoherent mixture of $\Omega$ states. Comparison between the numerical results and the DIS model reveals that $a$ is also not simply a weighted average over the single-potential reso- 
nances $a_{\Omega}$ and that the resonances in $a$ cannot be assigned to a single $\Omega$ state. Although the DIS approach assumes a coherent mixture of $\Omega$ states, coupling between the quasibound states is not taken into account, and it therefore does not describe the $\mathrm{Ne}^{*}$ system accurately. The overall behavior of $a$ is similar to that of the usual single-potential scattering length: neither the probability for encountering a positive nor a large value of $a$ is enhanced by the presence of five instead of one resonances.

The presence of an induced dipole-dipole interaction leads to strong coupling between the different $\Omega$ states and causes a broadening of the resonances, resulting in quasibound states that are a linear combination of different $\left|J \Omega P M_{P}\right\rangle$ states. This coupling between the different $\left|J \Omega P M_{P}\right\rangle$ states in turn leads to the inhomogeneous shift of the resonance positions and widths in the quantummechanical calculation as compared to the DIS approach. However, the dependence of the resonance positions and widths on the input potentials is quite straightforward. The resonance positions vary either directly proportional to the relative phase differences between the $\Omega$ potentials or not at all, depending on the exact composition of its quasibound state. The width of the strongly coupled resonances (whose positions vary $\propto-\Delta \phi$ ) is determined by the coupling to the ingoing channel, which varies periodically with $\Delta \phi$. The width of the weakly coupled resonances (whose positions do not vary with $\Delta \phi$ ) is constant, since the coupling to the ingoing channel does not change. The total change in all five resonance positions is always equal to $-\Delta \phi$ and the total width is conserved.

The possibility to choose between the two bosonic isotopes of $\mathrm{Ne}^{*}$ to optimize the value of the elastic cross section, greatly enhances the prospects for achieving BEC with $\mathrm{Ne}^{*}$ (Table II). Large beam fluxes of both bosonic isotopes, crucial in obtaining favorable initial conditions for efficient evaporative cooling, have been realized at the Eindhoven experiment [26], therefore choosing the isotope with the most favorable scattering length is feasible.

\section{ACKNOWLEDGMENTS}

We would like to thank S. Kotochigova for calculating the $a b$ initio potentials of spin-polarized $\mathrm{Ne}^{*}$ and S. Kokkelmans for careful reading of the manuscript. This work was financially supported by the Netherlands Foundation on Fundamental Research and Matter (FOM).
[1] M. Anderson et al., Science 269, 198 (1995).

[2] K.B. Davis et al., Phys. Rev. Lett. 75, 3969 (1995).

[3] C.C. Bradley, C.A. Sackett, J.J. Tollet, and R.G. Hulet, Phys. Rev. Lett. 75, 1687 (1995).

[4] G. Modugno et al., Science 294, 1320 (2001).

[5] T.C. Killian et al., Phys. Rev. Lett. 81, 3807 (1998).

[6] D.G. Fried et al., Phys. Rev. Lett. 81, 3811 (1998).

[7] A. Robert et al., Science 292, 461 (2001).

[8] F.P. Dos-Santos et al., Phys. Rev. Lett. 86, 3459 (2001).

[9] M. Zinner, P. Spoden, T. Kraemer, G. Birkl, and W. Ertmer, Phys. Rev. A 67, 010501 (2003).

[10] S.J.M. Kuppens et al., Phys. Rev. A 65, 023410 (2002).

[11] M.R. Doery, E.J.D. Vredenbregt, S.S. Op de Beek, H.C.W. Beijerinck, and B.J. Verhaar, Phys. Rev. A 58, 3673 (1998).

[12] G.V. Shlyapnikov, J.T.M. Walraven, U.M. Rahmanov, and M.W. Reynolds, Phys. Rev. Lett. 73, 3247 (1994).

[13] P.O. Fedichev, M.W. Reynolds, U.M. Rahmanov, and G.V. Shlyapnikov, Phys. Rev. A 53, 1447 (1996).

[14] V. Venturi, I.B. Whittingham, P.J. Leo, and G. Peach, Phys. Rev. A 60, 4635 (1999).

[15] V. Venturi and I.B. Whittingham, Phys. Rev. A 61, 060703(R) (2000).
[16] A. Derevianko and A. Dalgarno, Phys. Rev. A 62, 062501 (2000).

[17] O.J. Luiten, M.W. Reynolds, and J.T.M. Walraven, Phys. Rev. A 53, 381 (1996).

[18] B.J. Verhaar, J.M.V.A. Koelman, H.T.C. Stoof, O.J. Luiten, and S.B. Crampton, Phys. Rev. A 35, 3825 (1987).

[19] H.T.C. Stoof, J.M.V.A. Koelman, and B.J. Verhaar, Phys. Rev. B 38, 4688 (1988).

[20] S. Kotochigova (private communication).

[21] S. Kotochigova, E. Tiesinga, and I. Tupitsyn, Phys. Rev. A 61, 042712 (2000).

[22] G.F. Gribakin and V.V. Flambaum, Phys. Rev. A 48, 546 (1993).

[23] J.M. Vogels, B.J. Verhaar, and R.H. Blok, Phys. Rev. A 57, 4049 (1998).

[24] B.J. Verhaar, K. Gibble, and S. Chu, Phys. Rev. A 48, R3429 (1993).

[25] A.J. Moerdijk, W.C. Stwalley, R.G. Hulet, and B.J. Verhaar, Phys. Rev. Lett. 72, 40 (1994).

[26] J.G.C. Tempelaars, R.J.W. Stas, P.G.M. Sebel, H.C.W. Beijerinck, and E.J.D. Vredenbregt, Eur. Phys. J. D 18, 113 (2002). 